\title{
Finding Optimal Addition Chains Using a Genetic Algorithm Approach
}

\author{
Nareli Cruz-Cortés \\ Francisco Rodríguez-Henríquez \\ Raúl Juárez Morales \\ Carlos A. Coello Coello \\ Computer Science Section, Electrical Engineering Department \\ Centro de Investigación y de Estudios Avanzados del IPN \\ Av. Instituto Politécnico Nacional No. 2508, México D.F. \\ nareli@computacion.cs.cinvestav.mx, \{francisco, coello\}@cs.cinvestav.mx
}

\begin{abstract}
Since most public key cryptosystem primitives require the computation of modular exponentiation as their main building block, the problem of performing modular exponentiation efficiently has received considerable attention over the years. It is known that optimal (shortest) addition chains are the key mathematical concept for accomplishing modular exponentiations optimally. However, finding an optimal addition chain of length $r$ is an NP-hard problem whose search space size is comparable to $r$ !. In this contribution we explore the usage of a Genetic Algorithm (GA) approach for the problem of finding optimal (shortest) addition chains. We explain our GA strategy in detail reporting several promising experimental results that suggest that evolutionary algorithms may be a viable alternative to solve this illustrious problem in a quasi optimal fashion.
\end{abstract}

\section{Introduction}

Argueably, the field or modular exponentiation is the most important single arithmetic operation in public key cryptosystems. The search for efficient algorithm solutions for this problem has a long history whose roots can be traced as far back as the ancient works of Hindu mathematicians in 200 B.C [10]. In addition to its historical and theoretical relevance, field exponentiation has many important practical applications in the areas of error-correcting codes and cryptography. Modular exponentiation is used in several major public-key cryptosystems such as RSA, Diffie-Hellman and DSA [11]. For instance, the RSA crypto-scheme is based on the computation of $M^{e} \bmod n$, where $e$ is a fixed number, $M$ is an arbitrarily chosen numeric message and $n$ is the product of two large primes, namely, $n=p q$. Typical bit-lengths for $n$ used in commercial applications range from 1024 up to 4096 bits. In addition, modular exponentiation is also a major building block for several number theory problems including integer prime testing, integer factorization, field multiplicative inverse computation, etc.

Let $\alpha$ be an arbitrary integer in the range $[1, n-1]$, and $e$ and arbitrary positive integer. Then, we define modular exponentiation as the problem of finding 
the unique integer $\beta \in[1, n-1]$ that satisfies the equation

$$
\beta=\alpha^{e} \bmod n
$$

In order to improve legibility, in the rest of this paper we will drop the modular operator whenever it results unambiguous.

In general, there exist two main strategies for computing Equation (1) efficiently. One approach is to implement modular multiplication, the main building block required for modular exponentiation, as efficiently as possible. The other is to reduce the total number of multiplications needed to compute $\beta$. In this paper we address the latter approach, assuming that arbitrary choices of the base element $\alpha$ are allowed but with the restriction that the exponent $e$ is fixed.

The problem of determining the correct sequence of multiplications required for performing a modular exponentiation can be elegantly formulated by using the concept of addition chains. Formally, An addition chain for $e$ of length $l$ is a sequence $U$ of positive integers, $u_{0}=1, u_{1} \ldots, u_{l}=e$ such that for each $i>1$, $u_{i}=u_{j}+u_{k}$ for some $j$ and $k$ with $0 \leq j \leq k<i$. Therefore, if $U$ is an addition chain that computes $e$ as mentioned above, then for any $\alpha \in[1, n-1]$ we can find $\beta=\alpha^{e} \bmod n$ by successively computing: $\alpha, \alpha^{u_{1}}, \ldots, \alpha^{u_{l-1}}, \alpha^{e}$.

Let $l(e)$ be the shortest length of any valid addition chain for a given positive integer $e$. Then the theoretical minimum number of field multiplications required for computing the modular exponentiation of $(1)$ is precisely $l(e)$. Unfortunately, the problem of determining an addition chain for $e$ with the shortest length $l(e)$ is an NP-hard problem [11]. Therefore we have to use some sort of heuristic strategy in order to find an optimal addition chain when dealing with sufficiently large exponents $e$.

Generally speaking, a heuristic strategy tries to find in a reasonable time near optimal results for hard optimization problems, i.e. those problems having huge search spaces. Typically, a heuristic method starts from a non-optimal solution population and iteration after iteration improves its findings until a reasonable and/or valid solution can be achieved. The gradual improvement on the partial results is done using either deterministic or probabilistic search criteria. Given a fixed set of initial conditions, the optimized solutions obtained by a deterministic heuristic will remain unchanged from run to run. On the contrary, repeated executions of a probabilistic heuristic may produce different final solutions.

Across the centuries, a vast amount of algorithms for computing modular exponentiation have been reported. Reported strategies include: binary, m-ary, adaptive m-ary, power tree, the factor method, etc. [9-11].

On the other hand, relatively few probabilistic heuristics have been reported so far for finding near optimal addition chains $[13,4,3]$. In [13] a genetic algorithm search engine was proposed for solving this optimization problem but authors' strategy was only tested for small exponents. In [4] it was proposed the use of an artificial immune system as a probabilistic heuristic for finding minimal-length addition chains. Those optimal addition chains were then used for computing multiplicative inverses on binary extension fields. Although the addition chains found there were theoretically minimal, the required exponent sizes for that application are small (typically less than 10 bits). 
During the last three decades the interest on biologically inspired computing systems has grown in an important way $[12,7,6]$. Evolutionary Algorithms are perhaps the most well-known techniques from this group $[8,1]$, and they have been successfully applied to solve a very broad variety of optimization problems. In this paper, we present a Genetic Algorithm (GA) suited to optimize addition chains. The results obtained suggest that this approach is a very competitive alternative to the solution of the problem.

The rest of this paper is organized as follows. In Section 2 the problem in hand is stated in a formal way. Then, in Section 3 some well-known deterministic strategies for computing modular exponentiation are briefly described. In Section 4 the Genetic Algorithm approach used in this work is described in detail. Section 5 presents several experimental results obtained using our GA approach comparing them against the ones obtained by several selected deterministic strategies. Finally, in Section 6, our concluding remarks are drawn.

\section{Problem Statement}

The problem addressed in this work consists of finding the shortest addition chain for an exponent $e$. Formally, an addition chain can be defined as follows,

Definition An addition chain $U$ for a positive integer $e$ of length $l$ is a sequence of positive integers $U=\left\{u_{0}, u_{1}, \cdots, u_{l}\right\}$, and an associated sequence of $r$ pairs $V=\left\{v_{1}, v_{2} \cdots, v_{l}\right\}$ with $v_{i}=\left(i_{1}, i_{2}\right), 0 \leq i_{2} \leq i_{1}<i$, such that:

$-u_{0}=1$ and $u_{l}=e$

- for each $u_{i}, 1 \leq i \leq l, u_{i}=u_{i_{1}}+u_{i_{2}}$.

The search space for computing optimal addition chains increments its size at a factorial rate as there exist $r$ ! different and valid addition chains with length $r$. Clearly, the problem of finding the shortest ones becomes more and more complicated as $r$ grows larger.

\section{Deterministic Heuristics for Modular Exponentiation}

In this section, we briefly review some deterministic heuristics proposed in the literature for computing field exponentiation. For a complete description of these and other methods, interested readers are referred to [10,2].

Let $e$ be an arbitrary $m$-bit positive integer $e$, with a binary expansion representation given as, $e=\left(1 e_{m-2} \ldots e_{1} e_{0}\right)_{2}=2^{m-1}+\sum_{i=0}^{m-2} 2^{i} e_{i}$. Then,

$$
y=x^{e}=x^{\sum_{i=0}^{m-1} 2^{i} e_{i}}=\prod_{i=0}^{m-1} x^{2^{i} e_{i}}=\prod_{e_{i} \neq 0} x^{2^{i}}
$$

Binary strategies evaluate equation (2) by scanning the bits of the exponent $e$ one by one, either from left to right (MSB-first binary algorithm) or from right to left (LSB-first binary algorithm) applying Horner's rule. Both strategies require 
a total of $m-1$ iterations. At each iteration a squaring operation is performed, and if the value of the scanned bit is one, a subsequent field multiplication is performed. Therefore, the binary strategy requires a total of $m-1$ squarings and $H(e)-1$ field multiplications, where $H(e)$ is the Hamming weight of the binary representation of $e$. Thus, the computational complexity of the binary algorithm is given as,

$$
P(e, m)=m+H(e)-2=\left\lfloor\log _{2}(e)\right\rfloor+H(e)-1
$$

The binary method discussed above can be generalized by scanning more than one bit at a time. Hence, the window method (first described in [10]) scans $k$ bits at a time. The window method is based on a $k$-ary expansion of the exponent, where the bits of the exponent $e$ are divided into $k$-bit words or digits. The resulting words of $e$ are then scanned performing $k$ consecutive squarings and a subsequent multiplication as needed. For $k=1,2,3,4$ the window method is called, respectively, binary, quaternary octary and hexa MSB-first exponentiation method.

\section{The Proposed Genetic Algorithm}

In this work, we present a Genetic Algorithm (GA) approach suited for finding optimal addition chains. When applying a GA strategy it results crucial to select an appropriate chromosome representation as well as a well-defined fitness function. Additionally, any GA approach is instrumented by applying two main operators, namely, crossover and mutation operators. In the rest of this Section we describe how these design decisions were taken.

\subsection{Representation}

One of the most difficult decisions that must be taken when designing a GA is to select the most appropriate type of representation to encode the potential solutions of the problem of interest.

In this work, we adopt an integer encoding, using variable-length chromosomes. Each element from the addition chain is directly mapped on each gene in the chromosome. Then, in this case, the genotype and the phenotype are both the same.

For example, if we are minimizing the addition chain for the exponent $e=$ 6271 , one candidate solution could be

$1 \rightarrow 2 \rightarrow 4 \rightarrow 8 \rightarrow 10 \rightarrow 20 \rightarrow 30 \rightarrow 60 \rightarrow 90 \rightarrow 180 \rightarrow 360 \rightarrow 720 \rightarrow 1440 \rightarrow$ $2880 \rightarrow 5760 \rightarrow 5970 \rightarrow 6150 \rightarrow 6240 \rightarrow 6270 \rightarrow 6271$

This integer sequence represents a chromosome or individual $I$, where each gene $I_{k}$ corresponds to one step on the addition chain, for $0 \leq k \leq l$ with length $l=20$, and $I_{l}=6271$. 


\subsection{Fitness Function}

Since we are looking for the minimal addition chain's length, then the individual's fitness is precisely the addition chain's length, or in other words, the chromosome's length. The shorter the chromosome's length is, the better its fitness value, and vice versa.

For example, consider the following addition chain,

$1 \rightarrow 2 \rightarrow 4 \rightarrow 8 \rightarrow 10 \rightarrow 20 \rightarrow 30 \rightarrow 60 \rightarrow 90 \rightarrow 180 \rightarrow 360 \rightarrow 720 \rightarrow$ $1440 \rightarrow 2880 \rightarrow 5760 \rightarrow 5970 \rightarrow 6150 \rightarrow 6240 \rightarrow 6270 \rightarrow 6271$. $l=20$.

The associated fitness of this chain is equal to 20 because its length is precisely

\subsection{Crossover Operator}

The crossover operator creates two children from two parents. In our GA, we adopt one point crossover. Some extra considerations must be taken, however, mainly because of two reasons: first, it is necessary to assure that the resulting children are valid addition chains (i.e., feasible ones) and second, the chromosomes are of variable length. The way this operator produces offspring is illustrated in Figure 1 and it is defined in the following pseudocode,

\section{Begin Function Crossover}

For a pair of parents ( $P 1$ and $P 2)$ do:

1. Select a randomly selected crossover point $p$ such that $(2 \leq p \leq l-2)$ where $l$ is the chromosome length.

2. Create child $(C 1)$, copy the P1's values to the $\mathrm{C} 1$ starting from 0 until $p$ is reached, hence,

For $(k=0)$ to $(k=p) C 1_{k} \leftarrow P 1_{k}$

From the point $p$ until $e$ is reached, complete the child $\mathrm{C} 1$ following the rules by which P2 was created, in the following way,

For $(k=p+1)$ to $(k=$ length $)$

- Look for $a$ and $b$ values such that, $P 2_{k}=P 2_{a}+P 2_{b}$

- Set $C 1_{k} \leftarrow C 1_{a}+C 1_{b}$

EndFor

3. Create child $(C 2)$ :

For $(k=0)$ to $(k=p)$

$-C 2_{k} \leftarrow P 2_{k}$

EndFor

For $(k=p+1)$ to $(k=$ length $)$

- Look for $a$ and $b$ values such that, $P 1_{k}=P 1_{a}+P 1_{b}$

- Set $C 2_{k} \leftarrow C 2_{a}+C 2_{b}$

EndFor

\section{End Function Crossover}

We point out that the crossover operator is applied in the way described above only when that data manipulation does not produce values exceeding the exponent $e$. In case the value indicated by the crossover operator exceeds $e$ then the value assigned would be the maximum allowable. 


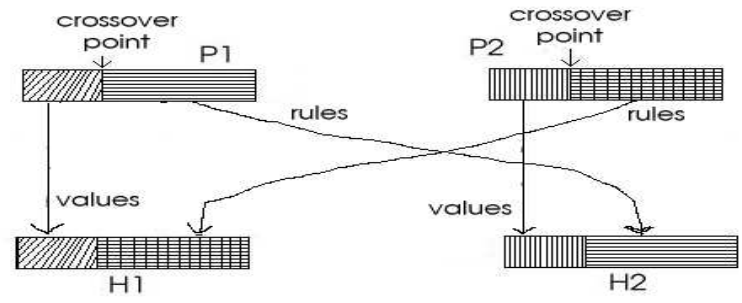

Fig. 1. Crossover Operator.

\subsection{Mutation Operator}

Next, we explain how the mutation operator was defined for this genetic algorithm. It is noted that our definition of this operator allows us to introduce random changes into the chromosome, while preserving addition chains' validity.

\section{BEGIN Function Mutation}

For each child $(C)$ do:

1. Randomly select a mutation point $i$ and a random number $j$ such that $2 \leq j<$ $i<(l-2)$, where $l$ is the chromosome length.

2. The new value of the child at the mutation point $C_{i+1}$ will be $C_{i+1}=C_{i}+C_{j}$

3. Repair the upper part of the chromosome $\left\{C_{k>i+1}\right\}$, using the following criterion: For $k=i+2$ to $l$, with $C_{l}=e$ do

If $(F l i p(Z))$ then use the doubling rule whenever is possible, i.e, $C_{k}=2 C_{k-1}$ Else if (flip(0.5)) set $C_{k}=C_{k-1}+C_{k-2}$

Else set $C_{k}=C_{m}+C_{n}$, where $m$ and $n$ are two randomly selected integers such that $0 \leq m, n<l$.

\section{END Function Mutation}

$\operatorname{Flip}(Z)$ is a function that receives an input parameter $Z$ such that $0 \leq Z \leq 1$. It returns true with probability $Z$, or false in other case.

\section{General GA}

Having defined the main Genetic Algorithm primitives, we proceed to put them together into the skeleton structure of the GA strategy outlined below,

\section{BEGIN-General-GA}

1. Randomly create an initial population size $N$.

2. cont $\leftarrow 0$

3. Repeat:

(a) Compute individuals' fitness.

(b) Select the $N$ parents to be reproduced.

(c) With probability $P c$, apply crossover operator to the $N$ parents so that a population of $N$ children is created.

(d) Apply the mutation operator to the children with a probability $\mathrm{Pm}$.

(e) Children will form the next generation population.

(f) cont $\leftarrow$ cont +1 
4. Go to step 3.(a) until cont $=$ Generations

5. Report the fittest individual.

END-General-GA.

\section{Experiments and Results}

In order to validate the GA approach described in the previous Section, we conducted a series of experiments, with the aim of comparing our GA's experimental results against the ones obtained by using several traditional deterministic methods.

The first set of experiments consisted on finding the accumulated addition chain lengths for all exponents $e$ in a given interval as it was done in [2]. Then, as a second test, we applied our genetic algorithm to a special class of exponents whose optimal addition chains are particularly hard to find. All our experiments were performed by applying the following GA's parameters: Population size $N=100$, Number of Generations = 300, Crossover Rate $P c=0.6$, Mutation Rate $P m=0.5$, Probability $Z=0.7$ (used in the mutation operator), Selection $=$ Binary Tournament. All the statistical results shown here were produced from 30 independent runs of the algorithm with different and independent random seeds (adopting a uniform distribution).

Using our genetic algorithm approach, we computed the accumulated addition chain lengths for all the first 1000 exponents, i.e. $e \in[1,1000]$. The accumulated value so obtained was then compared against the accumulated values reported in [2] by applying the following deterministic methods: Dyadic, Total, Fermat, Dichotomic, Factor, Quaternary and Binary $[10,2]$. All results found are shown in Table 1 . It can be seen that in the best case, our GA approach obtained better results than all the other six methods. In average, the GA approach was ranked in second place, only behind the Total method. It is noted that none of the features strategies was able to find the optimal value that was found by performing an exhaustive search.

Table 1. Accumulated addition chain lengths for exponents $e \in[1,1000]$

\begin{tabular}{|c|c|}
\hline \multicolumn{2}{|c|}{ Optimal value $=\mathbf{1 0 8 0 8}$} \\
\hline \hline Strategy & Total length \\
\hline Dyadic [2] & 10837 \\
\hline Total [2] & 10821 \\
\hline Fermat [2] & 10927 \\
\hline Dichotomic [2] & 11064 \\
\hline Factor [2] & 11088 \\
\hline Binary & 11925 \\
\hline Quaternary & 11479 \\
\hline Genetic Algorithm & Best: 10818 \\
& $\begin{array}{c}\text { Average: } 10824.07 \\
\text { Averagen: } 10824 \\
\end{array}$ \\
& Worst: 10830 \\
& Std.Dev.: 2.59 \\
\hline
\end{tabular}

Furthermore, we computed the accumulated addition chain lengths for all the exponents in the ranges $e \in[1,512], e \in[1,2000]$ and $e \in[1,4096]$. The methods used were the GA strategy, the binary method and the quaternary method. Once again and 
for comparison purposes, we computed the corresponding optimal values (obtained by enumeration). Those results are shown in Table 2 .

Clearly, the results obtained by the GA strategy outperformed both, the binary and the quaternary method, even in the worst case. We can observe that for the three cases considered (i.e., 512, 2000 and 4096), the GA obtained a reasonably good approximation of the optimal value.

Table 2. Accumulated addition chain lengths for 512, 2000 and 4096

\begin{tabular}{|c||c||c|}
\hline for all $e \in[1,512]$ & for all $e \in[1,2000]$ & for all $e \in[1,4096]$ \\
\hline Optimal: 4924 & Optimal: 24063 & Optimal: 54425 \\
\hline Binary: 5388 & Binary: 26834 & Binary: 61455 \\
\hline Quaternary: 5226 & Quaternary: 25923 & Quaternary: 58678 \\
\hline Genetic Algorithm & Genetic Algorithm & Genetic Algorithm \\
Best: 4925 & Best: 24124 & Best: 54648 \\
Average: 4927.7 & Average: 24135.17 & Average: 54684.13 \\
Averagen: 4927 & Averagen: 24136 & Averagen: 54685 \\
Worst: 4952 & Worst: 24144 & Worst: 54709 \\
Std.Dev.: 4.74 & Std.Dev: 5.65 & Std.Dev.: 13.55 \\
\hline
\end{tabular}

\section{A special class of exponents hard to optimize}

Let $e=c(r)$ be the smallest exponent that can be reached using an addition chain of length $r$. Solutions for that class of exponents are known up to $r=30$ and a compilation of them can be found in [5]. Interesting enough, the computational difficulty of finding shortest addition chains for those exponents seems to be among the hardest if not the hardest one from the studied exponent families [10]. We show the solutions found by the GA for the class of exponents shown in Table 3. It is noted that in 24 out of 30 exponents, the GA approach was able to find the shortest addition chain. However, for the 6 remaining exponents (namely, 357887, 1176431, 2211837,4169527, 7624319 and 14143037), our GA strategy found addition chains that where one unit above the optimal.

Notice that the searching space size for this special class of exponents (considering both feasible and infeasible individuals) is $r$ !. Hence, in the case of $r=30$, finding the shortest addition chain for the exponent $c(r=30)=14143037$, implied to launch a searching effort over a search space size of about,

$r !=30 !=265252859812191058636308480000000 \approx 2^{107}$ possibilities.

\section{Conclusions and Future Work}

In this paper we described how a genetic algorithm strategy can be applied to the problem of finding shortest addition chains for optimal field exponentiation computations. The GA heuristic presented in this work was capable of finding almost all the optimal addition chains for any given fixed exponent $e$ with $e<4096$. Taking into account the optimal value (which was found by enumeration) the percentage error of our GA strategy was within $0.4 \%$ from the optimal for all cases considered. In other words, for any given fixed exponent $e$ with $e<4096$, our strategy was able to find the requested shortest addition chain in at least $99.6 \%$ of the cases. In a second experiment 
for assessing the actual power of the GA strategy as a search engine, we tested it for generating the shortest addition chains of a class of exponents particularly hard to optimize, whose optimal lengths happen to be known for the first 30 members of the family. In most cases considered, the GA strategy was able to find the optimal values. Future work includes finding quasi optimal addition chains for 128-bit exponents and beyond such as the ones typically used in commercial cryptographic applications.

\section{Acknowledgments}

The first and second authors acknowledge support from CONACyT through the CONACyT project number 45306. The third and fourth authors acknowledge support from CONACyT through the NSF-CONACyT project number 42435-Y.

\section{References}

1. Thomas Bäck, David B. Fogel, and Zbigniew Michalewicz, editors. Handbook of Evolutionary Computation. Institute of Physics Publishing and Oxford University Press, New York, 1997.

2. F. Bergeron, J. Berstel, and S. Brlek. Efficient computation of addition chains. Journal de thorie des nombres de Bordeaux, 6:21-38, 1994.

3. J. Bos and M. Coster. Addition chain heuristics. In G. Brassard, (editor) Advances in Cryptology —CRYPTO 89 Lecture Notes in Computer Science, 435:400-407, 1989.

4. N. Cruz-Cortes, F.. Rodriguez-Henriquez, and C. Coello Coello. On the optimal computation of finite field exponentiation. In C Lematre, C. Reyes, J. Gonzlez, (editors) Advances in Artificial Intelligence - IBERAMIA 2004: 9th Ibero-American Conference on AI Lecture Notes in Computer Science, 3315:747-756, November 2004.

5. D. Bleinchenbacher and A. Flammenkamp. An Efficient Algorithm for Computing Shortest Addition Chains, 1997.

6. Leandro Nunes de Castro and Jonathan Timmis. An Introduction to Artificial Immune Systems: A New Computational Intelligence Paradigm. Springer-Verlag, 2002.

7. M. Dorigo and G. Di Caro. The Ant Colony Optimization Meta-Heuristic. In D. Corne, M. Dorigo, and F. Glover, editors, New Ideas in Optimization. McGrawHill, 1989.

8. David E. Goldberg. Genetic Algorithms in Search, Optimization and Machine Learning. Addison-Wesley Publishing Co., Reading, Massachusetts, 1989.

9. D. M. Gordon. A survey of fast exponentiation methods. Journal of Algorithms, 27(1):129-146, April 1998.

10. Donald Ervin Knuth. The Art of Computer Programming 3rd. ed. Addison-Wesley, Reading, Massachusetts, 1997.

11. A. J. Menezes, Paul C. van Oorschot, and Scott A.Vanstone. Handbook of Applied Cryptography. CRC Press, Boca Raton, Florida, 1996.

12. A. Michalewicz and D. Fogel. How to Solve It: Modern Heuristics. Springer, 1996.

13. N. Nedjah and LD. Mourelle. Efficient pre-processing for large window-based modular exponentiation using genetic algorithms. In Developments in Applied Artificial Intelligence Lecture Notes in Artificial Intelligence, 2718:625-635, 2003. 
Table 3. Shortest addition chains for a special class of exponents

\begin{tabular}{|c|c|c|}
\hline $\begin{array}{c}\text { exponent } \\
e=c(r)\end{array}$ & Addition Chain & $\begin{array}{c}\text { Length } \\
\mathrm{r}\end{array}$ \\
\hline 1 & 1 & 0 \\
\hline 2 & $1 \rightarrow 2$ & 1 \\
\hline 3 & $1 \rightarrow 2 \rightarrow 3$ & 2 \\
\hline 5 & $1 \rightarrow 2 \rightarrow 3 \rightarrow 5$ & 3 \\
\hline 7 & $1 \rightarrow 2 \rightarrow 3 \rightarrow 5 \rightarrow 7$ & 4 \\
\hline 11 & $1 \rightarrow 2 \rightarrow 3 \rightarrow 5 \rightarrow 8 \rightarrow 11$ & 5 \\
\hline 19 & $1 \rightarrow 2 \rightarrow 3 \rightarrow 5 \rightarrow 7 \rightarrow 12 \rightarrow 19$ & 6 \\
\hline 29 & $1 \rightarrow 2 \rightarrow 3 \rightarrow 4 \rightarrow 7 \rightarrow 11 \rightarrow 18 \rightarrow 29$ & 7 \\
\hline 47 & $1 \rightarrow 2 \rightarrow 3 \rightarrow 5 \rightarrow 10 \rightarrow 20 \rightarrow 40 \rightarrow 45 \rightarrow 47$ & 8 \\
\hline 71 & $1 \rightarrow 2 \rightarrow 3 \rightarrow 5 \rightarrow 7 \rightarrow 12 \rightarrow 17 \rightarrow 34 \rightarrow 68 \rightarrow 71$ & 9 \\
\hline 127 & $1 \rightarrow 2 \rightarrow 4 \rightarrow 6 \rightarrow 12 \rightarrow 24 \rightarrow 48 \rightarrow 72 \rightarrow 120 \rightarrow 126 \rightarrow 127$ & 10 \\
\hline 191 & $1 \rightarrow 2 \rightarrow 3 \rightarrow 5 \rightarrow 10 \rightarrow 20 \rightarrow 21 \rightarrow 42 \rightarrow 63 \rightarrow 126 \rightarrow 189 \rightarrow 191$ & 11 \\
\hline 379 & $1 \rightarrow 2 \rightarrow 4 \rightarrow 5 \rightarrow 10 \rightarrow 15 \rightarrow 25 \rightarrow 50 \rightarrow 75 \rightarrow 150 \rightarrow 300 \rightarrow 375 \rightarrow 379$ & 12 \\
\hline 607 & $1 \rightarrow 2 \rightarrow 4 \rightarrow 6 \rightarrow 12 \rightarrow 24 \rightarrow 48 \rightarrow 96 \rightarrow 192 \rightarrow 384 \rightarrow 576 \rightarrow 600 \rightarrow 606 \rightarrow 607$ & 13 \\
\hline 1087 & $\begin{aligned} 1 \rightarrow 2 \rightarrow 3 \rightarrow 6 \rightarrow 12 \rightarrow 18 \rightarrow 36 \rightarrow & 74 \rightarrow 144 \rightarrow 216 \rightarrow 432 \rightarrow 864 \rightarrow 865 \rightarrow 1081 \\
& \rightarrow 1087\end{aligned}$ & 14 \\
\hline 1903 & $\begin{aligned} 1 \rightarrow 2 \rightarrow 3 \rightarrow 5 \rightarrow 10 \rightarrow 13 \rightarrow 26 & \rightarrow 52 \rightarrow 104 \rightarrow 105 \rightarrow 210 \rightarrow 420 \rightarrow 840 \rightarrow 1680 \\
& \rightarrow 1890 \rightarrow 1903\end{aligned}$ & 15 \\
\hline 3583 & $\begin{aligned} 1 \rightarrow 2 \rightarrow 3 \rightarrow 6 \rightarrow 12 \rightarrow 18 \rightarrow & 36 \rightarrow 72 \rightarrow 108 \rightarrow 216 \rightarrow 432 \rightarrow 864 \rightarrow 1728 \rightarrow 3456 \\
& \rightarrow 3564 \rightarrow 3582 \rightarrow 3583\end{aligned}$ & 16 \\
\hline 6271 & $\begin{array}{c}1 \rightarrow 2 \rightarrow 3 \rightarrow 6 \rightarrow 12 \rightarrow 24 \rightarrow 48 \rightarrow 96 \rightarrow 192 \rightarrow 384 \rightarrow 768 \rightarrow 1536 \rightarrow 3072 \rightarrow 6144 \\
\rightarrow 6240 \rightarrow 6264 \rightarrow 6270 \rightarrow 6271\end{array}$ & 17 \\
\hline 11231 & $\begin{aligned} 1 \rightarrow 2 \rightarrow 3 \rightarrow 6 \rightarrow 12 & \rightarrow 24 \rightarrow 25 \rightarrow 50 \rightarrow 100 \rightarrow 200 \rightarrow 400 \rightarrow 800 \rightarrow 1600 \rightarrow 3200 \\
\rightarrow 6400 & \rightarrow 9600 \rightarrow 11200 \rightarrow 11225 \rightarrow 11231\end{aligned}$ & 18 \\
\hline 18287 & $\begin{aligned} 1 \rightarrow 2 \rightarrow 3 \rightarrow 6 \rightarrow 9 & \rightarrow 15 \rightarrow 30 \rightarrow 45 \rightarrow 47 \rightarrow 94 \rightarrow 188 \rightarrow 190 \rightarrow 380 \rightarrow 760 \rightarrow 1520 \\
& \rightarrow 3040 \rightarrow 6080 \rightarrow 12160 \rightarrow 18240 \rightarrow 18287\end{aligned}$ & 19 \\
\hline 34303 & $\begin{aligned} 1 \rightarrow 2 \rightarrow 3 & \rightarrow 6 \rightarrow 12 \rightarrow 14 \rightarrow 28 \rightarrow 56 \rightarrow 112 \rightarrow 224 \rightarrow 448 \rightarrow 504 \rightarrow 1008 \rightarrow 2016 \\
& \rightarrow 4032 \rightarrow 8064 \rightarrow 16128 \rightarrow 32256 \rightarrow 34272 \rightarrow 34300 \rightarrow 34303\end{aligned}$ & 20 \\
\hline 65131 & $\begin{aligned} 1 \rightarrow 2 & \rightarrow 3 \rightarrow 6 \rightarrow 12 \rightarrow 24 \rightarrow 48 \rightarrow 72 \rightarrow 144 \rightarrow 288 \rightarrow 576 \rightarrow 1152 \rightarrow 2304 \rightarrow 4608 \\
& \rightarrow 4611 \rightarrow 9222 \rightarrow 18444 \rightarrow 27666 \rightarrow 55332 \rightarrow 55908 \rightarrow 65130 \rightarrow 65131\end{aligned}$ & 21 \\
\hline 110591 & $\begin{aligned} & \rightarrow 1 \rightarrow 2 \rightarrow 4 \rightarrow 5 \rightarrow 10 \rightarrow 20 \rightarrow \\
& \rightarrow 2570 \rightarrow 5140 \rightarrow 70 \rightarrow 80 \rightarrow 160 \rightarrow 320 \rightarrow 640 \rightarrow 1280 \rightarrow 2560 \\
& \rightarrow 7710 \rightarrow 12850 \rightarrow 25700 \rightarrow 51400 \rightarrow 102800 \rightarrow 110510 \rightarrow 110590 \\
& \rightarrow 110591\end{aligned}$ & 22 \\
\hline 196591 & $\begin{aligned} 1 \rightarrow 2 \rightarrow 3 \rightarrow 6 & \rightarrow 12 \rightarrow 15 \rightarrow 30 \rightarrow 60 \rightarrow 120 \rightarrow 240 \rightarrow 480 \rightarrow 720 \rightarrow 1440 \rightarrow 2880 \\
\rightarrow 5760 \rightarrow 11520 & \rightarrow 23040 \rightarrow 46080 \rightarrow 92160 \rightarrow 184320 \rightarrow 19584 \rightarrow 196560 \rightarrow 196590 \\
& \rightarrow 196591\end{aligned}$ & 23 \\
\hline 357887 & $\begin{array}{c}1 \rightarrow 2 \rightarrow 3 \rightarrow 4 \rightarrow 8 \rightarrow 16 \rightarrow 32 \rightarrow 64 \rightarrow 128 \rightarrow 256 \rightarrow 257 \rightarrow 514 \rightarrow 771 \rightarrow 11542 \\
\rightarrow 3084 \rightarrow 6168 \rightarrow 12336 \rightarrow 24672 \rightarrow 49344 \rightarrow 49347 \rightarrow 98691 \rightarrow 148038 \rightarrow 296076 \\
\rightarrow 345423 \rightarrow 357759 \rightarrow 357887\end{array}$ & 24 \\
\hline 685951 & $\begin{aligned} 1 \rightarrow 2 \rightarrow 4 & \rightarrow 6 \rightarrow 7 \\
\rightarrow 3360 & \rightarrow 6720 \rightarrow 14 \rightarrow 21 \rightarrow 42 \rightarrow 84 \rightarrow 168 \rightarrow 336 \rightarrow 504 \rightarrow 840 \rightarrow 1680 \\
& \rightarrow 13440 \rightarrow 26880 \rightarrow 53760 \rightarrow 57120 \rightarrow 114240 \rightarrow 228480\end{aligned}$ & 25 \\
\hline 1176431 & $\begin{aligned} 1 \rightarrow 2 & \rightarrow 4 \rightarrow 5 \rightarrow 10 \rightarrow 15 \rightarrow 19 \rightarrow 38 \rightarrow 76 \rightarrow 152 \rightarrow 304 \rightarrow 608 \rightarrow 612 \\
\rightarrow 1224 & \rightarrow 2448 \rightarrow 4896 \rightarrow 9792 \rightarrow 19584 \rightarrow 29376 \rightarrow 58752 \rightarrow 117504 \rightarrow 235008 \\
\rightarrow & \rightarrow 352512 \rightarrow 587520 \rightarrow 1175040 \rightarrow 1176264 \rightarrow 1176416 \rightarrow 1176431\end{aligned}$ & 27 \\
\hline 2211837 & $\begin{array}{c}1 \rightarrow 2 \rightarrow 3 \rightarrow 6 \rightarrow 9 \rightarrow 15 \rightarrow 30 \rightarrow 60 \rightarrow 120 \rightarrow 126 \rightarrow 252 \rightarrow 504 \rightarrow 1008 \\
\rightarrow 2016 \rightarrow 4032 \rightarrow 8062 \rightarrow 16128 \rightarrow 16143 \rightarrow 32286 \rightarrow 64572 \rightarrow 129144 \rightarrow 258288 \\
\rightarrow 516576 \rightarrow 1033152 \rightarrow 2066304 \rightarrow 2195448 \rightarrow 2211591 \rightarrow 2211717 \rightarrow 2211837\end{array}$ & 28 \\
\hline 4169527 & $\begin{array}{c}1 \rightarrow 2 \rightarrow 3 \rightarrow 6 \rightarrow 12 \rightarrow 24 \rightarrow 48 \rightarrow 96 \rightarrow 192 \rightarrow 384 \rightarrow 768 \rightarrow 1536 \rightarrow 2304 \\
\rightarrow 4608 \rightarrow 9216 \rightarrow 18432 \rightarrow 36864 \rightarrow 73728 \rightarrow 147456 \rightarrow 294912 \rightarrow 589824 \rightarrow 589825 \\
\rightarrow 1179650 \rightarrow 1769475 \rightarrow 3538950 \rightarrow 4128775 \rightarrow 4165639 \\
\rightarrow 414167943 \rightarrow 4169479 \rightarrow 4169527\end{array}$ & 29 \\
\hline \begin{tabular}{|l|}
7624319 \\
\end{tabular} & $\begin{aligned} 1 \rightarrow 2 \rightarrow 3 \rightarrow 6 & \rightarrow 12 \rightarrow 18 \rightarrow 36 \rightarrow 72 \rightarrow 144 \rightarrow 288 \rightarrow 576 \rightarrow 1152 \rightarrow 1224 \\
\rightarrow & \rightarrow 2448 \rightarrow 4896 \rightarrow 9792 \rightarrow 19584 \rightarrow 39168 \rightarrow 78336 \rightarrow 156672 \rightarrow 313344 \\
& \rightarrow 626688 \rightarrow 1253376 \rightarrow 1254600 \rightarrow 1274184 \rightarrow 1274185 \rightarrow 2548370 \\
& \rightarrow 5096740 \rightarrow 6370925 \rightarrow 7624301 \rightarrow 7624319\end{aligned}$ & 30 \\
\hline 14143037 & $\begin{array}{c}1 \rightarrow 2 \rightarrow 3 \rightarrow 6 \rightarrow 12 \rightarrow 18 \rightarrow 30 \rightarrow 60 \rightarrow 120 \rightarrow 240 \rightarrow 480 \rightarrow 960 \rightarrow 961 \\
\rightarrow 1922 \rightarrow 3844 \rightarrow 7688 \rightarrow 11532 \rightarrow 23064 \rightarrow 46128 \rightarrow 92256 \rightarrow 184512 \rightarrow 369024 \\
\rightarrow 461280 \rightarrow 830304 \rightarrow 1660608 \rightarrow 3321216 \rightarrow 6642432 \\
\rightarrow 13284864 \rightarrow 14115168 \rightarrow 14138232 \rightarrow 14142076 \rightarrow 14143037\end{array}$ & 31 \\
\hline
\end{tabular}

